\title{
Fluctuation analysis of lung function as a predictor of long-term response to
} $\beta_{2}$-agonists

\author{
C. Thamrin*, G. Stern*, M-P.F. Strippoli”, C.E. Kuehni", B. Suki", \\ D.R. Taylor ${ }^{+}$and U. Frey*
}

ABSTRACT: The response to $\boldsymbol{\beta}_{2}$-agonists differs between asthmatics and has been linked to subsequent adverse events, even death. Possible determinants include $\beta_{2}$-adrenoceptor genotype at position 16, lung function and airway hyperresponsiveness. Fluctuation analysis provides a simple parameter $\alpha$ measuring the complex correlation properties of day-to-day peak expiratory flow. The present study investigated whether $\alpha$ predicts clinical response to $\beta_{2}$-agonist treatment, taking into account other conventional predictors.

Analysis was performed on previously published twice-daily peak expiratory flow measurements in 66 asthmatic adults over three 6-month randomised order treatment periods: placebo, salbutamol and salmeterol. Multiple linear regression was used to determine the association between $\alpha$ during the placebo period and response to treatment (change in the number of days with symptoms), taking into account other predictors namely $\beta_{2}$-adrenoceptor genotype, lung function and its variability, and airway hyperresponsiveness.

The current authors found that $\alpha$ measured during the placebo period considerably improved the prediction of response to salmeterol treatment, taking into account genotype, lung function or its variability, or airway hyperresponsiveness.

The present study provides further evidence that response to $\beta_{2}$-agonists is related to the time correlation properties of lung function in asthma. The current authors conclude that fluctuation analysis of lung function offers a novel predictor to identify patients who may respond well or poorly to treatment.

KEYWORDS: Asthma, $\beta_{2}$-adrenoceptor genotype, bronchodilators, fractals, peak expiratory flow

$\mathbf{T}$ he $\beta_{2}$-agonist controversy is based on substantial evidence relating $\beta_{2}$-agonist use to adverse asthma outcomes, including mortality [1-5]. Responses to $\beta_{2}$-agonists differ among individuals $[2,6]$. This heterogeneity may partly be related to $\beta_{2}$-adrenoceptor (ADRB2) genotypes [2, 6-12]. The homozygous Arg-16 polymorphism has been associated with poorer outcomes $[7,8]$, although findings are not entirely consistent $[13,14]$. Additionally, airway hyperresponsiveness, lung function and its variability have been proposed to predict treatment response [15]. However, often only a weak relationship exists between these factors and treatment response, suggesting that conventional pathophysiological measurements are inadequate to predict the behaviour of complex diseases such as asthma [16, 17]. Therefore, a crucial target for investigation remains: to find new predictors that allow clinicians to identify patients who would or would not benefit from treatment.

The complexities in asthma could be characterised in a novel way, by considering it as a dynamical disease [16, 18-23]. In this context, the likelihood of loss of asthma control may be better characterised by fluctuation analysis [24] applied to a time series of daily variations in peak expiratory flow (PEF) [18]. The method yields a single parameter $\alpha$, which quantifies the strength of long-range correlations present in the time series. The presence of long-range correlations means that PEF on any given day is dependent on its values on previous days, even over longer time intervals [18], consistent with fractal behaviour. Hence, $\alpha$ can be thought of as a measure of

\section{AFFLLIATIONS}

*Division of Respiratory Medicine, Dept of Paediatrics, Inselspital, University of Bern, and \#Institute of Social and Preventive Medicine, University of Bern, Bern, Switzerland.

"Dept of Biomedical Engineering, Boston University, Boston, MA, USA. +Faculty of Medicine, Dunedin School of Medicine, University of Otago, Dunedin, New Zealand.

CORRESPONDENCE

C. Thamrin

Division of Paediatric Respiratory Medicine,

Dept of Paediatrics, University of Bern, Inselspital,

3010 Bern, Switzerland.

Fax: 41316324807

E-mail: cindy.thamrin@insel.ch

Received:

July 142008

Accepted after revision:

October 082008

\section{SUPPORT STATEMENT}

C. Thamrin was supported by the European Respiratory Society

Fellowship Grant 80 (Lausanne, Switzerland) and the Roche Research Foundation (Basel, Switzerland). C.E. Kuehni received Swiss National Science Foundation (Bern, Switzerland) PROSPER grants 3233069348 and $3200-069349$.

STATEMENT OF INTEREST None declared. 
complexity arising from the intrinsic control of the system producing the fluctuations, and may be influenced by external stimuli. Fluctuation analyses have been applied to heart rate variability [24], end-tidal oxygen and carbon dioxide concentrations [25], and tidal volume [25, 26]. Analysis of heart rate fluctuations has even been used to predict tachycardia following a myocardial infarction [27]. Using twice-daily $\mathrm{PEF}$, it has been reported that in a group of mild-to-moderate asthmatic adults in a randomised placebo-controlled three-way crossover study [28], $\alpha$ tended to increase, and thus asthma control improved, during long-term salmeterol treatment. In contrast, $\alpha$ significantly decreased during long-term salbutamol treatment [18]. Thus, $\alpha$ has value in characterising asthma control during treatment.

In the present study, the current authors aimed to determine whether $\alpha$ measured in the absence of regular treatment, using data obtained during the placebo period $(\alpha(P L))$, has value in predicting clinical response to long-term $\beta_{2}$-agonist treatment. To answer this, the association between $\alpha(\mathrm{PL})$ and changes in the number of days with clinical symptoms during treatment was determined, taking into account other important predictors of treatment response, namely ADRB2 genotype, lung function and its variability, and airway hyperresponsiveness.

\section{MATERIALS AND METHODS}

\section{Study population and design}

The current study was a retrospective analysis of a randomised, double-blind, double-dummy, crossover study, approved by the Otago and Canterbury ethics committees (New Zealand) [28, 29]. Briefly, 157 mild-to-moderate asthmatic adult subjects underwent a 4-week run-in period involving assessment of spirometric lung function (forced expiratory volume in one second (FEV1)) and airway responsiveness to methacholine (provocative concentration causing a 20\% fall in FEV1 (PC20)). This was followed by three 6-month randomised treatment periods, during which subjects received salmeterol $50 \mu \mathrm{g}$ twice daily, salbutamol $400 \mu \mathrm{g}$ four times daily, or lactose placebo, with intervening 4-week placebo washout periods. Subjects maintained the same dosage of their inhaled corticosteroids, if applicable, for $\geqslant 3$ months prior to and during the present study, but were allowed on-demand rescue bronchodilators and emergency oral corticosteroids for exacerbations as appropriate.

Patients were instructed to record their PEF and respiratory symptoms twice daily on a diary card. Symptoms recorded included day- and night-time chest tightness/wheeze/dyspnoea, cough, sputum production, exercise and nocturnal wakening, rated on a 0 to 3 scale or a yes/no response where applicable. A composite asthma score, taking into account symptoms, morning PEF and rescue bronchodilator use, was computed for each study day, ranging from 0 for stable asthma to 4 for major exacerbation/medical emergency. Details of the asthma score calculation have been previously published [28].

Of the 157 subjects in the database ( 80 of whom have had their fluctuation analysis data reported previously [18]), 66 were identified who had undergone genotyping for ADRB2 position 16 [29], lung function and airway hyperresponsivess testing, and for whom fluctuation analysis [18] data were available in all three treatment periods.

\section{Fluctuation analysis}

Fluctuation analysis was undertaken using custom-written software (Matlab; The Mathworks Inc., Natick, MA, USA), as described previously [18]. The analysis was limited to PEF time series with 300 data points for each treatment period, corresponding to the first 150 days of each treatment period, in order to standardise the data length across subjects. The time series was first integrated and then divided into nonoverlapping windows of size $\mathrm{n}$. The local trend in each window was removed by fitting and subtracting a regression line from the integrated data. The root-mean-square values of the detrended signal were calculated for a given window length $\mathrm{n}$ to yield the detrended fluctuation function $(\mathrm{F}(\mathrm{n}))$. This calculation was then repeated for increasing $n$, and $\log F(n)$ was plotted against $\log n$. Typically, $F(n)$, a measure of the fluctuations, increases with n. A linear relation between log $\mathrm{F}(\mathrm{n})$ versus $\log \mathrm{n}$ indicates the presence of scaling, which can be characterised by the slope $\alpha$ of the regression line fit.

A PEF time series with $\alpha$ of 0.5 indicates a system that is not deterministic and is prone to instabilities and exacerbations, whereas higher $\alpha$ values imply more deterministic behaviour with stronger correlations present; hence they are more likely to be the expression of stable and more predictable asthma control [18].

\section{Clinical outcome}

From the asthma score the total number of symptom days were derived, i.e. days when the asthma score was $>0$ within the same 150-day period, which was used for fluctuation analysis. The difference in the number of symptom days between placebo and either the salbutamol or salmeterol treatment period (i.e. treatment minus placebo days) was the primary outcome. Thus, an increase (positive difference) in the number of symptom days indicates deterioration in clinical condition, while a decrease (negative difference) indicates improvement. This outcome was less directly dependent on PEF than the asthma score (which included morning PEF as one dimension).

\section{Statistical analysis}

Multiple linear regression models were used to examine associations between potential predictors of interest and clinical outcome. To test the robustness of the current findings, two sensitivity analyses were performed using slightly different statistical approaches. First, the number of symptom days during treatment per se (i.e. not the difference from placebo) were examined using a negative binomial regression model instead of linear regression, because the number of symptom days was not normally distributed but conformed to that expected from count or rate data. The more conventional Poisson regression (a special case of negative binomial regression) was not appropriate due to overdispersion, i.e. high variance compared with the mean. Secondly, the linear regression was repeated using differences in the mean asthma score between placebo and treatment as outcome, instead of differences in the number of symptom days.

A standard model was defined as the model in which adjustments were made for age, sex, treatment order and the number of symptom days during the placebo period. The possible effect of treatment order was adjusted for using the position of the relevant treatment period within the sequence 


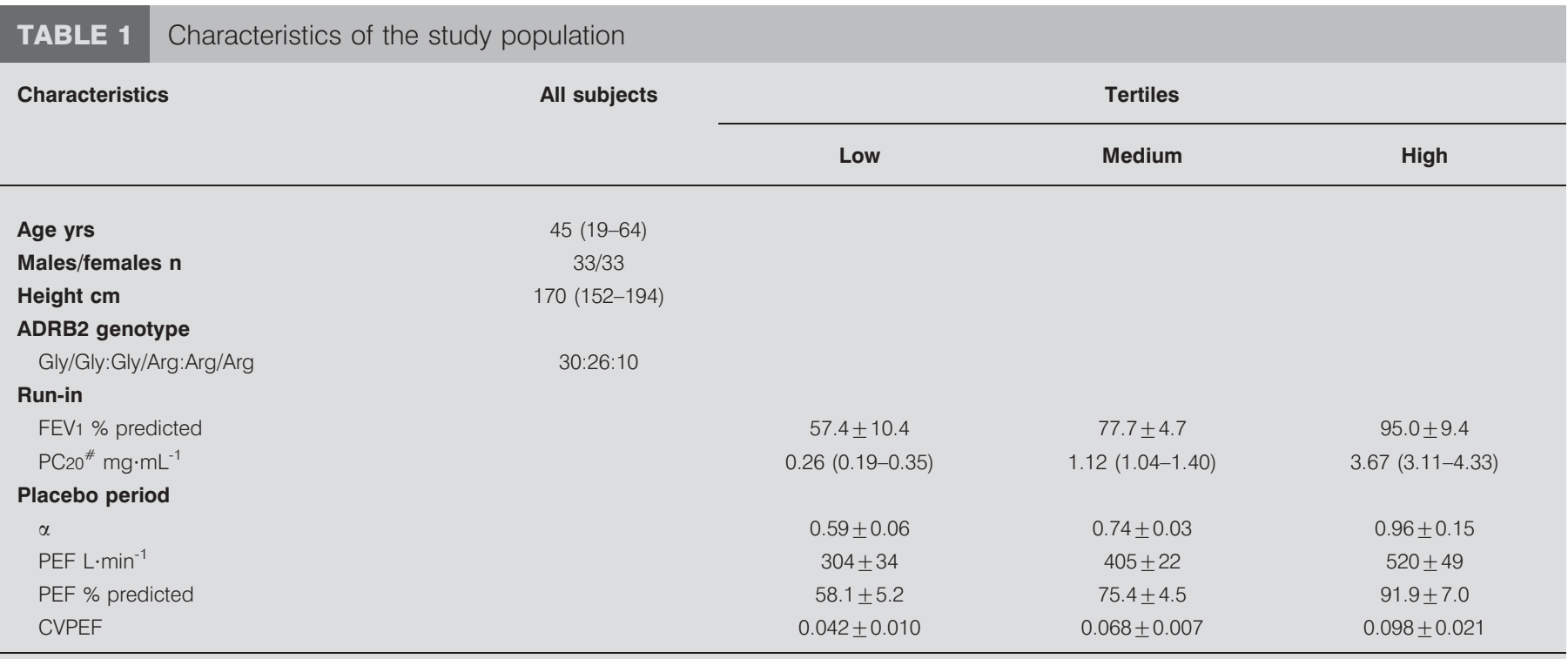

Data are presented as median (range) and mean \pm SD, unless otherwise stated. ADRB2: $\beta_{2}$-adrenoceptor; FEV1: forced expiratory volume in one second; PC20: provocative concentration causing a $20 \%$ fall in FEV 1 ; PEF: peak expiratory flow; CVPEF: coefficient of variation of PEF. ${ }^{*}$ : geometric mean with $95 \%$ confidence intervals. ADRB2 genotype, lung function and airway hyperresponsiveness parameters were obtained during the run-in period prior to treatment randomisation (FEV1 and $\mathrm{PC} 20)$. $\alpha$, PEF and CVPEF were calculated from PEF during the placebo period; see Methods section for full descriptions of parameters.

of treatments. All continuous, (i.e. noncategorical) variables, such as age and number of symptom days during the placebo period, were centred to the mean.

In a first, simple model, potential predictors of outcome were examined separately by adding each respective predictor to the standard model. Predictors of interest were: $\alpha(\mathrm{PL})$; ADRB2 genotype; lung function expressed as a percentage of the predicted value for the subject [30] (PEF \% pred, FEV1\% pred); coefficient of variation of PEF (CVPEF); and airway hyperresponsiveness (PC20). To facilitate interpretation, these were stratified into low, medium and high tertiles, and a comparison was made with the lowest tertile as baseline, except for the ADRB2 genotype, for which the Gly/Gly genotype was used as the reference group. Thus, the coefficients reported are relative to the baseline group of subjects with low $\alpha(\mathrm{PL})$, low PEF \% pred, low FEV1\% pred, etc., with the baseline group effect represented by the constant term of the regression.

Predictors that were found to be significantly $(p<0.05)$ associated with the outcome were then included into a fully adjusted, multivariable model. Potential interaction between $\alpha(\mathrm{PL})$ and genotype within the multivariable model was tested for statistical significance using the F-test.

To evaluate how well $\alpha(\mathrm{PL})$ predicted the response to treatment relative to other parameters, the adjusted $r^{2}$ value of the standard model was compared with models which included one or more of the predictors of interest $(\alpha(\mathrm{PL})$, ADRB2 genotype, PEF \% pred and CVPEF during the placebo period, and $\mathrm{FEV}_{1} \%$ pred and $\mathrm{PC}_{20} \%$ pred during run-in). Predictors were tested for significance using the F-test.

\section{Sensitivity to PEF quality control criteria}

For fluctuation analysis, 300 PEF data points (150 days) were used with $<3 \%$ missing data. The present authors investigated the effect of relaxing these strict quality control criteria for inclusion of PEF time series into the study (by using shorter PEF series or allowing a greater percentage of missing data). Details and results are found in the Appendix.

\section{RESULTS}

\section{Subject characteristics}

Characteristics, spirometric lung function and airway hyperresponsiveness of the 66 subjects obtained at run-in (FEV1 and $\mathrm{PC} 20)$, as well as potential predictors obtained during the placebo periods $(\alpha(\mathrm{PL}), \mathrm{PEF}$ and $\mathrm{CVPEF})$, are summarised in table 1 . The mean \pm SD values of the low, medium and high tertiles for the predictors are also shown. The three genotype groups were: homozygous Gly-16 (Gly/Gly); heterozygous (Gly/Arg); and homozygous Arg-16 (Arg/Arg). The genotype frequencies in the original population have been shown to be consistent with the Hardy-Weinberg equilibrium [29]. Clinical assessment parameters, i.e. the mean asthma score and number of symptom days during the placebo and treatment periods are given in table 2 .

\section{Association of $\alpha(P L)$ with clinical response to treatment}

For salmeterol, $\alpha(\mathrm{PL}), \mathrm{PEF} \%$ pred during placebo period and Arg/Arg genotype were significantly associated with response to treatment, both in the simple and the multivariable regression models (table 3 ). The association between $\alpha(\mathrm{PL})$ and change in days with symptoms during salmeterol treatment became stronger in the multivariable model, with a mean improvement of -17.9 and -16.1 days with symptoms in individuals with medium and high $\alpha(\mathrm{PL})$, respectively, compared with those with low values. The association with genotype was not significant for Gly/Arg $(p=0.278)$ and was weak for $\operatorname{Arg} / \operatorname{Arg}(p=0.044)$. There was no evidence of an interaction between $\alpha(\mathrm{PL})$ and genotype in the current data $(\mathrm{p}=0.739$ for interaction). 
TABLE 2 Asthma score and number of days with symptoms during the placebo, salbutamol and salmeterol treatment periods

\begin{tabular}{lccc} 
& Placebo & Salbutamol & Salmeterol \\
\hline $\begin{array}{l}\text { Mean asthma score } \\
\text { Median number of days with symptoms }\end{array}$ & $\begin{array}{ccc}0.15(0.05-0.33) \\
22(7-46)\end{array}$ & $\begin{array}{c}0.15(0.09-0.39) \\
22(12-56)\end{array}$ & $0.05(0.01-0.13)^{+}$ \\
\hline
\end{tabular}

Data are presented as median (interquartile range). Outcomes were calculated over an observation period of 150 days; $\mathrm{n}=66 .{ }^{*}$ : mean over the observation period of a composite asthma score comprising symptoms, morning peak expiratory flow and rescue bronchodilator use computed for each study day, ranging from 0 for stable asthma to 4 for major exacerbation/medical emergency; ": median over the observation period for the number of days on which a non-0 asthma score was recorded; ${ }^{+}$ statistical significance (Wilcoxon signed rank test, $\mathrm{p}<0.05$ ) compared with placebo.

For salbutamol, the only predictor that was significantly associated with treatment outcome was PEF \% pred during the placebo period, in both the simple and multivariable regression models (see online supplementary material table E1).

The changes in the number of symptom days in response to both salbutamol and salmeterol are illustrated in figure 1 . With increasing $\alpha(\mathrm{PL})$ from the low, medium to high tertiles, relatively small change in symptom days was observed with salbutamol while progressively fewer symptom days were observed with salmeterol.

\section{Comparison of $\alpha(P L)$ with other predictors}

The $\mathrm{r}^{2}$ values of the standard regression model as well as models including one or more of the predictors of interest are shown in table 4 . Adding $\alpha(\mathrm{PL})$ to the model improved the

TABLE 3 Association between patient characteristics at baseline and response to salmeterol treatment (defined as change in number of symptom days from the placebo period)

\begin{tabular}{|c|c|c|c|c|c|c|}
\hline \multirow[t]{2}{*}{ Effect } & \multicolumn{3}{|c|}{ Simple model } & \multicolumn{3}{|c|}{ Multivariable ${ }^{\#}$} \\
\hline & Coefficient & $95 \% \mathrm{Cl}$ & p-value & Coefficient & $95 \% \mathrm{Cl}$ & p-value \\
\hline \multicolumn{7}{|l|}{$\alpha(\mathrm{PL})^{\bullet}$} \\
\hline Medium & -15.3 & $-27.4--3.1$ & 0.015 & -17.9 & $-29.4--6.4$ & 0.003 \\
\hline High & -15.4 & $-28.2--2.7$ & 0.018 & -16.1 & $-28.5--3.8$ & 0.012 \\
\hline \multicolumn{7}{|c|}{ ADRB2 genotype } \\
\hline Gly/Arg & -2.8 & $-14.3-8.8$ & 0.633 & -5.6 & $-16.0-4.7$ & 0.278 \\
\hline Arg/Arg & -17.2 & $-32.9--1.4$ & 0.034 & -14.6 & $-28.9--0.4$ & 0.044 \\
\hline \multicolumn{7}{|c|}{ PEF \% pred during placebo period } \\
\hline Medium & 11.6 & $-1.0-24.3$ & 0.071 & 8.3 & $-3.3-19.9$ & 0.158 \\
\hline High & 17.6 & $4.1-31.1$ & 0.012 & 22.9 & $10.4-35.4$ & 0.001 \\
\hline \multicolumn{7}{|c|}{ CVPEF during placebo period } \\
\hline Medium & -1.5 & $-14.8-11.7$ & 0.815 & & & \\
\hline High & -11.2 & $-26.9-4.8$ & 0.158 & & & \\
\hline \multicolumn{7}{|c|}{$\mathrm{FEV}_{1} \%$ pred } \\
\hline Medium & 7.7 & $-5.1-20.6$ & 0.234 & & & \\
\hline High & 16.1 & $2.9-29.3$ & 0.018 & & & \\
\hline \multicolumn{7}{|l|}{$\mathrm{PC}_{20}$} \\
\hline Medium & 2.6 & $-10.9-16.1$ & 0.702 & & & \\
\hline High & 6.4 & $-7.0-19.9$ & 0.343 & & & \\
\hline Constant $^{+}$ & & & & -1.5 & $-15.5-12.4$ & 0.825 \\
\hline
\end{tabular}

Results were derived from multiple linear regression. All models were adjusted for age, sex, sequence of treatment and number of symptom days during placebo period by default. All noncategorical variables such as age and number of symptom days during the placebo period were centred to the corresponding mean. Comparisons were made against having low values of $\alpha$ during placebo $(\alpha(\mathrm{PL})$ ), Gly/Gly genotype and low peak expiratory flow (PEF) \% pred, coefficient of variation of PEF (CVPEF) during the placebo period, forced expiratory volume in one second (FEV1) \% pred and provocative concentration causing a $20 \%$ fall in $\mathrm{FEV}_{1}$ (PC20) during the run-in period. Cl: confidence interval; ADRB2: $\beta_{2}$-adrenocepter. ${ }^{*}: r^{2}=0.677$ for the fully-adjusted multivariable model. Due to collinearity, only one lung function parameter was included in the multivariable model. ${ }^{*}$ : if a patient had either a medium (or high) $\alpha(\mathrm{PL}$ ), then that patient would be likely to have had 18 (or 16 ) fewer days with symptoms during the 150 days of salmeterol treatment, i.e. $11 \%$ of treatment days, compared with the placebo period. ${ }^{+}$: a patient with Gly/Gly genotype, a low $\alpha$ and PEF \% pred during the placebo period would have a mean improvement in number of symptom days of 1.5 (1\%) days during the 150 days of salmeterol treatment compared with the placebo period. 
goodness-of-fit from 0.563 in the standard model to 0.604 . This was a similar improvement to adding PEF \% pred into the model, and better than for genotype or PC20. The goodness-offit increased to 0.662 when both $\alpha(\mathrm{PL})$ and PEF \% pred were included in the model, suggesting independent effects.

Similar results were obtained with the two alternative statistical approaches (predicting number of symptom days per se using a negative binomial model and predicting the difference in mean asthma score with a linear regression model). Results for genotype became weaker with asthma score as the outcome, but the associations with $\alpha(\mathrm{PL})$ and PEF $\%$ pred remained highly significant (see online supplementary material tables E2 and E3, respectively).

\section{DISCUSSION}

In the present study, the current authors set out to determine the clinical utility of fluctuation analysis of PEF and specifically whether it helps to predict treatment outcome over and above conventionally used predictors. $\alpha(\mathrm{PL})$ was found to be strongly and independently related to the change in clinical symptoms in response to salmeterol treatment. When coupled with PEF \% pred, $\alpha(\mathrm{PL})$ adds considerable predictive power to using CVPEF, FEV1 \% pred, PC20 and ADRB2 position 16 genotype.

\section{Interpretation of findings}

The strength of $\alpha$ as a predictor of treatment response lends further support to the idea that asthma is a complex dynamic disease [16, 18-21], well-characterised by a parameter that quantifies long-range correlations. In practical terms, if a patient has either a medium (or high) $\alpha$ during the placebo period, then that patient would be likely to have 18 (or 16) fewer days with symptoms during the 150 days of salmeterol treatment, i.e. $11 \%$ of treatment days. This compares with only

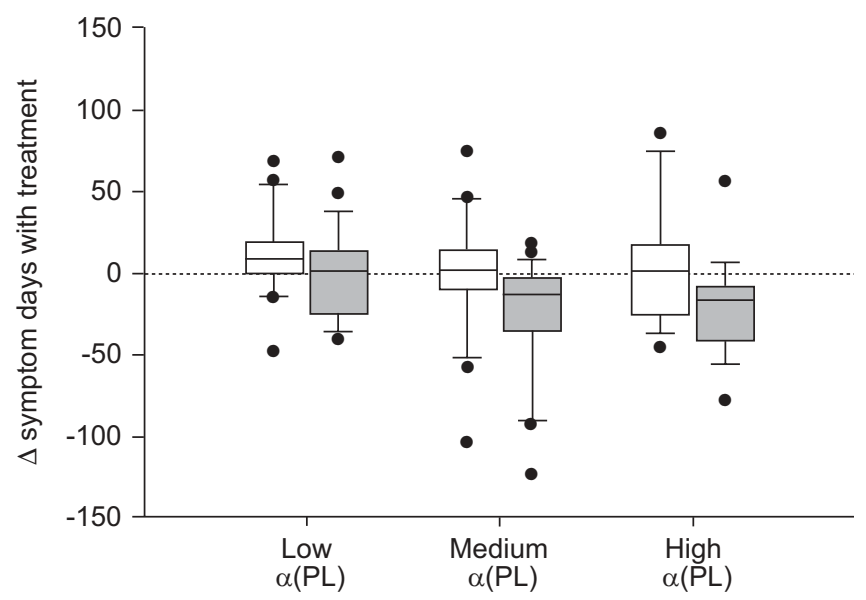

FIGURE 1. Box plots showing the change $(\triangle)$ in number of symptom days from placebo during treatment with salbutamol $(\square)$ and salmeterol ( $\square$ ), in subjects with values of $\alpha$ during placebo $(\alpha(P L))$ within the low, medium and high tertiles. The median is shown for each box plot, with the box boundaries denoting the 25th and 75th percentiles, and the error bars denoting the 10th and 90th percentiles. outliers. A negative change indicated improvement of symptoms due to decreased number of symptom days with treatment compared with placebo. With increasing $\alpha(\mathrm{PL})$ from the low, medium to high tertiles, relatively little changes in symptom days were seen with salbutamol and progressively less symptom days for salmeterol.
$1 \%$ (from the constant term in the regression) of treatment days in a patient with a low $\alpha$ and PEF \% pred (based on data from table 3 ). It is notable that a medium $\alpha$ appears to be as good as a high $\alpha$, as the high tertile does not appear to result in further improvement compared with the medium tertile [18].

Interestingly, if a patient had a high PEF \% pred during the placebo period, they would have $23(15 \%)$ more symptom days with treatment compared with low PEF \% pred, corrected for the number of symptom days during placebo. At first glance this seems counterintuitive, but it could be due to a "ceiling" effect, whereby a person with low PEF \% pred has greater room for improvement with treatment and thus a very large negative change in symptom days, while a person with high PEF \% pred has only a modest negative change in symptom days.

There was a lack of predictive power of $\alpha(\mathrm{PL})$ for outcomes during salbutamol treatment. A possible explanation, consistent with previous findings [18], is that the frequent short-term stimuli provided by salbutamol results in deterioration in asthma control independently of baseline $\alpha$. As a consequence, the PEF pattern becomes irregular in time, which in turn results in a significant loss of predictive power.

Combining $\alpha(\mathrm{PL})$ and PEF $\%$ pred explained almost $10 \%$ of the total goodness-of-fit in modelling individual responses to treatment. This is especially beneficial as it does not require an additional measurement procedure for the patient, since the two are calculated from the same peak flow measurements. It is worth making the distinction between $\alpha$ and PEF here. A high $\alpha$ is not the only criterion for stability, since it is an indicator of time correlation properties of the PEF, as opposed to the properties of the PEF magnitude distribution. A patient with a high $\alpha$, but low mean PEF or high CVPEF, may still have poorly controlled asthma, since these are independent properties of the daily PEF behaviour, both of which contribute independently to predicting whether or not a patient remains stable [20].

\section{Significance of findings}

In a previous study, the presence of long-range correlations in PEF was found, and $\alpha$ was useful in characterising long-term treatment response [18]. The present study shows that it is also useful in predicting long-term treatment response, even when measured in the absence of such treatment (in this case, during the placebo period). Also of note in the previous findings was the suggestion that there might be some "optimum" value of $\alpha$, since any positive or negative deviation in $\alpha$ from a mean of 0.78 during the placebo period corresponded to a tendency for $\alpha$ to return to 0.78 with treatment [18]. In the present study, more evidence of this is seen, although in this case a plateau is more apparent: improvement in symptoms was optimally predicted when $\alpha$ was in the medium or high tertiles. Additionally, using multiple regression the current authors were able to complement the value of $\alpha$ by taking into account other factors, notably PEF \% pred, and show that it compares favourably with other conventional predictors.

The present results add value to the use of daily peak flow measurements in assessing and monitoring patients with asthma [31] by looking at its variability in a new way. Previously, there has been doubt that regular measurements 
TABLE 4 Comparison of goodness-of-fit

\begin{tabular}{lcc}
\hline Potential predictor & Adjusted $^{\#} \mathbf{r}^{2}$ & p-value \\
\hline Standard model & 0.563 & \\
$\boldsymbol{\alpha}(\mathrm{PL})$ & 0.604 & 0.022 \\
ADRB2 genotype & 0.582 & 0.102 \\
PEF \% pred during placebo period & 0.599 & 0.030 \\
CVPEF during placebo period & 0.565 & 0.328 \\
FEV1\% pred & 0.590 & 0.059 \\
PC20 & 0.555 & 0.632 \\
$\boldsymbol{\alpha}(\mathrm{PL})$ and PEF \% pred during placebo period & 0.662 & 0.001 \\
Multivariable model (fully adjusted) & 0.677 & 0.001 \\
\hline
\end{tabular}

Comparison between linear regression models with different potential predictors of the change in number of symptom days from the placebo period with salmeterol treatment. All models were adjusted for age, sex, sequence of treatment and number of symptom days during placebo period by default. The predictors values of $\alpha$ during placebo $(\alpha(P L))$, peak expiratory flow (PEF) $\%$ pred, coefficient of variation of PEF (CVPEF), forced expiratory volume in one second ( $\left.F E V_{1}\right) \%$ pred and provocative concentration causing a $20 \%$ fall in FEV1 (PC20) were categorised into tertiles of low, medium and high values. For $\beta_{2}$ adrenocecptor (ADRB2) position 16 genotype, Gly/Gly was the reference group. ${ }^{*}$ : each parameter was added to the model separately, i.e. the adjusted $r^{2}$ values are not cumulative; " : p-values are shown for the F-test, where the null hypothesis was that the relevant parameter was equal to zero (i.e. not associated with the outcome).

of peak flow add value in monitoring asthma given that they do not appear to be consistently related to current symptoms [32-34]. ZHANG et al. [17] have pointed out that multiple measurements of lung function over a length of time are a minimum criterion for being able to establish end-points for asthma control. With the advent of electronic peak flow monitoring and appropriate software for analyses, their predictive role in assessing asthma control may deserve renewed attention. Furthermore, a multi-dimensional approach to monitoring asthma, taking into account various factors and their relative contributions to asthma, is increasingly being advocated $[16,20,32,35]$. The characterisation of time correlation properties of lung function complements this approach.

\section{Limitations and open questions}

The present study has a number of limitations. First, the numbers are small. One drawback of fluctuation analysis is that a large number of data points, e.g. 150 days, is necessary for the determination of long-range correlations. Very strict quality control criteria (see Appendix) were used to determine acceptability of individual PEF time series data into the current study. This restricted the total number of eligible patients from the original study. Relaxing these criteria was investigated but it was found that as the length of the PEF series decreased, the association of $\alpha(\mathrm{PL})$ with outcome became less significant compared with \% pred PEF. This is not surprising given that the interpretation of long-range correlations becomes less important when the time range of the data is decreased. Similarly, as the percentage of missing data increased, a similar but less pronounced pattern emerged. Thus, fluctuation analysis is to some extent dependent on the completeness of the data, and its use is limited to long-term monitoring of asthma. However, in such cases an extra dimension of usefulness was provided to data that would already be available [31]. Note that the actual time over which the data was collected was not the principal limitation of the method rather, it was the number of data points required to characterise the fluctuation dynamics. Analysis of variability from respiratory data collected with greater frequency and over shorter time-scales was performed in relation to asthma $[19,23,36]$, but the time-scale over which interpretation of the results was made would likely have to be adjusted accordingly. It may be that fluctuations over short time-scales can predict behaviour at longer time-scales, which would be a great advantage, but this has yet to be shown.

Secondly, corticosteroids and on-demand $\beta_{2}$-agonist bronchodilators were permitted throughout the current study. There is evidence that concurrent use of corticosteroids mitigates the adverse effect of long-acting $\beta_{2}$-agonists [37]. However, in the present study the contribution of $\alpha(\mathrm{PL})$ and PEF to predicting better or worse response to salmeterol was apparent even in the presence of corticosteroid use.

Thirdly, a past history of smoking was not adjusted for, which could be a potential confounder given recent evidence for the interaction between passive smoking and the ADRB2 genotype [38]. However, current and ex-smokers ( $>5$ pack-yrs) were excluded from the present study.

Finally, $\alpha$ was calculated using data obtained from the placebo period. Arguably, the run-in period would have been more suitable as it was prior to any treatment. Unfortunately, it was too short to allow adequate fluctuation analysis to be performed. However, both periods would be comparable, given that during the run-in the subjects were taking placebo, there was a washout of 4 weeks between treatment periods, and the possible effect of treatment order was adjusted for in the current regression analysis. Despite these limitations, what is clear from the present study is that the relationship between $\alpha(\mathrm{PL})$ and symptom response to treatment remained strongly significant using different adjustments, outcomes and regression models.

In conclusion, having previously identified the utility of longrange correlations in daily lung function for characterising $\beta_{2-}$ agonist treatment responses, the present authors here report their utility in predicting response to treatment. Fluctuation analysis of baseline lung function measurements was strongly associated with changes in symptoms with long-term treatment, in this instance using salmeterol. Baseline $\alpha$, when coupled with mean daily peak flows, added considerably to the prediction of outcome compared with other conventional single measures of spirometric lung function, variability of lung function, airway hyperresponsiveness, as well as the $\beta_{2^{-}}$ adrenoceptor genotype, and contained information that seemed to be distinct and independent of these factors. This novel approach of looking at the time history of peak expiratory flow recordings, distinct from studying its mean or variability, not only provides additional insight into asthma control but also offers a potential new parameter to predict whether a patient will respond favourably or adversely to treatment in the future. This is particularly important in light 
of the potential detrimental response to $\beta_{2}$-agonists. It also constitutes a step towards the multi-dimensional approach to asthma monitoring, which is increasingly valid in such a complex disease.

\section{APPENDIX: SENSITIVITY TO QUALITY CONTROL CRITERIA OF PEF TIME SERIES}

For the present study and in previous work, strict acceptability criteria was used to determine whether a time series was to be included in the analysis. The criteria were as follows: $\geqslant 300$ PEF data points (150 days) available, and $<3 \%$ missing data. Furthermore, these two criteria had to be met in all treatment and placebo periods for a subject to be included into the study. Where there were missing data, they were replaced by the PEF value of the previous corresponding day/night, as detailed previously [18].

The present authors repeated the regression analyses of tables 3 and 4, while comparing $\alpha(\mathrm{PL})$ calculated using the following criteria: using 300, 200 and 100 data points, and allowing $<3 \%$, $<5 \%$ and $<10 \%$ missing data in the PEF time series. To allow comparison across different data lengths, the outcome (change in number of symptom days from placebo to treatment period) was calculated over the entire 150-day observation period regardless of data length used to calculate $\alpha(\mathrm{PL})$.

The association of $\alpha(\mathrm{PL})$ with outcome was found to become less significant as the length of the PEF series decreased from 300 to 100 data points (from -14.2 days per observation period $(p=0.009)$ to -8.9 days per observation period $(p=0.096)$; both for the highest $\alpha(\mathrm{PL})$ tertile), as \% pred PEF during placebo period became more important. The association of $\alpha(\mathrm{PL})$ with outcome also became less significant as the percentage of missing data increased from $3 \%$ (from -14.2 days per observation period $(p=0.009)$ to -9.8 days per observation period ( $p=0.058$ ); both for the highest $\alpha(\mathrm{PL})$ tertile) to $10 \%$, although the effect was marginally less pronounced than with the data length. However, $\alpha(\mathrm{PL})$ and PEF \% pred still yielded the greatest increase in model fit from the standard model, regardless of data length or the amount of missing data.

Relaxing the criterion from 300 to 100 data points resulted in the percentage of acceptable subjects changing from 71 to only $72 \%$ out of the total subjects with otherwise complete data $(n=108)$. When relaxing the criterion from 3 to $10 \%$ missing data allowable, the percentage of acceptable subjects changed from 71 to $87 \%$.

\section{ACKNOWLEDGEMENTS}

The authors would like to thank G.P. Herbison (Dept of Preventive and Social Medicine, Dunedin School of Medicine, Dunedin, New Zealand) for assistance with the original data and codes, and P. Latzin (Paediatric Respiratory Medicine, Inselspital, Bern, Switzerland) and B. Spycher (Institute of Social and Preventive Medicine, University of Bern, Bern) for valuable feedback and discussion.

\section{REFERENCES}

1 Nelson HS, Weiss ST, Bleecker ER, Yancey SW, Dorinsky PM, the SMART Study Group. The salmeterol multicenter asthma research trial: a comparison of usual pharmacotherapy for asthma or usual pharmacotherapy plus salmeterol. Chest 2006; 129: 15-26.

2 Martinez FD. Serious adverse events and death associated with treatment using long-acting $\beta$-agonists. Clin Rev Allergy Immunol 2006; 31: 269-278.

3 Chinchilli VM. General principles for systematic reviews and meta-analyses and a critique of a recent systematic review of long-acting $\beta$-agonists. J Allergy Clin Immunol 2007; 119: 303-306.

4 Hasford J, Virchow JC. Excess mortality in patients with asthma on long-acting $\beta_{2}$-agonists. Eur Respir J 2006; 28: 900-902.

5 Cates CJ, Cates MJ. Regular treatment with salmeterol for chronic asthma: serious adverse events. Cochrane Database Syst Rev 2008; 3: CD006363.

6 Shore SA, Drazen JM. $\beta$-agonists and asthma: too much of a good thing? J Clin Invest 2003; 112: 495-497.

7 Israel E, Drazen JM, Liggett SB, et al. The effect of polymorphisms of the $\beta_{2}$-adrenergic receptor on the response to regular use of albuterol in asthma. Am J Respir Crit Care Med 2000; 162: 75-80.

8 Israel E, Chinchilli VM, Ford JG, et al. Use of regularly scheduled albuterol treatment in asthma: genotype-stratified, randomised, placebo-controlled cross-over trial. Lancet 2004; 364: 1505-1512.

9 Wechsler ME, Lehman E, Lazarus SC, et al. $\beta$-adrenergic receptor polymorphisms and response to salmeterol. Am J Respir Crit Care Med 2006; 173: 519-526.

10 Taylor DR. Pharmacogenetics of $\beta_{2}$-agonist drugs in asthma. Clin Rev Allergy Immunol 2006; 31: 247-258.

11 Martinez FD, Graves PE, Baldini M, Solomon S, Erickson R. Association between genetic polymorphisms of the $\beta_{2^{-}}$ adrenoceptor and response to albuterol in children with and without a history of wheezing. J Clin Invest 1997; 100: 3184-3188.

12 Taylor DR, Hall IP. ADRB2 polymorphisms and $\beta_{2}$ agonists. Lancet 2007; 370: 2075-2076.

13 Tattersfield AE, Harrison TW. $\beta$-adrenoceptor polymorphisms: focus moves to long-acting $\beta$-agonists. Am J Respir Crit Care Med 2006; 173: 473-474.

14 Bleecker ER, Postma DS, Lawrance RM, Meyers DA, Ambrose HJ, Goldman M. Effect of ADRB2 polymorphisms on response to longacting $\beta_{2}$-agonist therapy: a pharmacogenetic analysis of two randomised studies. Lancet 2007; 370: 2118-2125.

15 Tantisira KG, Fuhlbrigge AL, Tonascia J, et al. Bronchodilation and bronchoconstriction: predictors of future lung function in childhood asthma. J Allergy Clin Immunol 2006; 117: 1264-1271.

16 Frey U. Predicting asthma control and exacerbations: chronic asthma as a complex dynamic model. Curr Opin Allergy Clin Immunol 2007; 7: 223-230.

17 Zhang J, Yu C, Holgate ST, Reiss TF. Variability and lack of predictive ability of asthma end-points in clinical trials. Eur Respir J 2002; 20: 1102-1109.

18 Frey U, Brodbeck T, Majumdar A, et al. Risk of severe asthma episodes predicted from fluctuation analysis of airway function. Nature 2005; 438: 667-670.

19 Que CL, Kenyon CM, Olivenstein R, Macklem PT, Maksym GN. Homeokinesis and short-term variability of human airway caliber. J Appl Physiol 2001; 91: 1131-1141. 
20 Frey U, Suki B. Complexity of chronic asthma and chronic pulmonary disease: implications for disease progression and control. Lancet 2008; 372: 1088-1099.

21 Belair J, Glass L, An Der Heiden U, Milton J. Dynamical disease: identification, temporal aspects and treatment strategies of human illness. Chaos 1995; 5: 1-7.

22 Macklem PT. Can airway function be predicted? Am J Respir Crit Care Med 1996; 153: S19-S20.

23 Que CL, Maksym G, Macklem PT. Deciphering the homeokinetic code of airway smooth muscle. Am J Respir Crit Care Med 2000; 161: S161-S163.

24 Peng CK, Havlin S, Stanley HE, Goldberger AL. Quantification of scaling exponents and crossover phenomena in nonstationary heartbeat time series. Chaos 1995; 5: 82-87.

25 Cernelc M, Suki B, Reinmann B, Hall GL, Frey U. Correlation properties of tidal volume and end-tidal $\mathrm{O}_{2}$ and $\mathrm{CO}_{2}$ concentrations in healthy infants. J Appl Physiol 2002; 92: 1817-1827.

26 Baldwin DN, Suki B, Pillow JJ, Roiha HL, Minocchieri S, Frey U. Effect of sighs on breathing memory and dynamics in healthy infants. J Appl Physiol 2004; 97: 1830-1839.

27 Makikallio TH, Seppanen T, Airaksinen KE, et al. Dynamic analysis of heart rate may predict subsequent ventricular tachycardia after myocardial infarction. Am J Cardiol 1997; 80: 779-783.

28 Taylor DR, Town GI, Herbison GP, et al. Asthma control during long term treatment with regular inhaled salbutamol and salmeterol. Thorax 1998; 53: 744-752.

29 Taylor DR, Drazen JM, Herbison GP, Yandava CN, Hancox RJ, Town GI. Asthma exacerbations during long term $\beta$ agonist use: influence of $\beta_{2}$ adrenoceptor polymorphism. Thorax 2000; 55: 762-767.
30 Nunn AJ, Gregg I. New regression equations for predicting peak expiratory flow in adults. BMJ 1989; 298: 1068-1070.

31 Reddel HK. Peak flow monitoring in clinical practice and clinical asthma trials. Curr Opin Pulm Med 2006; 12: 75-81.

32 Global Initiative for Asthma (GINA). Global Strategy for Asthma Management and Prevention. http://www. ginasthma.org Date last accessed: October 8, 2008.

33 Brand PLP, Duiverman EJ, Waalkens HJ, van EssenZandvliet EEM, Kerrebijn KF. Peak flow variation in childhood asthma: correlation with symptoms, airways obstruction, and hyperresponsiveness during long-term treatment with inhaled corticosteroids. Dutch CNSLD Study Group. Thorax 1999; 54: 103-107.

34 Kerstjens HA, Brand PL, de Jong PM, Koeter GH, Postma DS. Influence of treatment on peak expiratory flow and its relation to airway hyperresponsiveness and symptoms. The Dutch CNSLD Study Group. Thorax 1994; 49: 1109-1115.

35 Fuhlbrigge AL. Asthma severity and asthma control: symptoms, pulmonary function, and inflammatory markers. Curr Opin Pulm Med 2004; 10: 1-6.

36 Diba C, Salome CM, Reddel HK, Thorpe CW, Toelle B, King GG. Short-term variability of airway caliber - a marker of asthma? J Appl Physiol 2007; 103: 296-304.

37 Bateman E, Nelson H, Bousquet J, et al. Meta-analysis: effects of adding salmeterol to inhaled corticosteroids on serious asthma-related events. Ann Intern Med 2008; 149: 33-42.

38 Zhang G, Hayden CM, Khoo SK, et al. $\beta_{2}$-adrenoceptor polymorphisms and asthma phenotypes: interactions with passive smoking. Eur Respir J 2007; 30: 1334-1341. 